\title{
Pricing of defaultable bonds with random information flow
}

\author{
Dorje C. Brody ${ }^{1,2}$ and Yan Tai Law ${ }^{3}$ \\ ${ }^{1}$ Mathematical Sciences, Brunel University, Uxbridge UB8 3PH, UK \\ ${ }^{2}$ Department of Optical Physics and Modern Natural Science, \\ St. Petersburg National Research University of Information Technologies, \\ Mechanics and Optics, 49 Kronverksky Avenue, St. Petersburg 197101, Russia \\ ${ }^{3}$ Department of Mathematics, Imperial College London, London SW7 2BZ, UK
}

(Dated: April 16, 2015)

In the information-based approach to asset pricing the market filtration is modelled explicitly as a superposition of signals concerning relevant market factors and independent noise. The rate at which the signal is revealed to the market then determines the overall magnitude of asset volatility. By letting this information flow rate random, we obtain an elementary stochastic volatility model within the information-based approach. Such an extension is justified on account of the fact that in real markets information flow rates are rarely measurable. Effects of having a random information flow rate is investigated in detail in the context of a simple model setup. Specifically, the price process of an elementary defaultable bond derived, and its characteristic behaviours are revealed via simulation studies. The price of a European-style option on the bond is worked out, showing that the model has a sufficient flexibility to fit volatility surface. As an extension of the random information flow model, modelling of price manipulation is considered. A simple model is used to show how the skewness of the manipulated and unmanipulated price processes take opposite signature.

\section{INTRODUCTION}

In the information-based asset pricing framework of Brody, Hughston and Macrina (hereafter the BHM framework) the starting point is the specification of a model for the market filtration, along with the cash flow of the asset [1-4]. The market filtration, more specifically, is generated by a market information process that takes the form of a superposition of a 'signal' component associated with the cash flow of the asset (or, more generally, market factors relevant to the actual cash flow) and an independent 'noise' component that obscures the value of the cash flow. The simplest model for the information process within the BHM framework was introduced in the context of modelling credit-risky discount bond price process $[1]$. Specifically, we fix a probability space $\left(\Omega, \mathcal{F}_{t}, \mathbb{Q}\right)$, where $\mathbb{Q}$ denotes the risk-neutral measure, and let $X_{T}$ denote the random variable representing the impending cash flow of a credit-risky bond, with maturity $T$. The process that generates market information is then defined by

$$
\xi_{t}=\sigma X_{T} t+\beta_{t T}
$$

where $\left\{\beta_{t T}\right\}_{0 \leq t \leq T}$ is a standard Brownian bridge on the interval $[0, T]$, independent of $X_{T}$, and information flow-rate parameter $\sigma>0$ is assumed constant.

The rationale of the model (1) can be described briefly as follows. Before time $T$ market participants do not have direct access to the value of the cash flow. Market participants 
nevertheless have partial information (signal) concerning the value of $X_{T}$, which according to this simple model choice is revealed to the market at a constant rate $\sigma$. This 'signal' is however obscured by an independent 'noise', which is modelled here by a Brownian bridge $\left\{\beta_{t T}\right\}$. The market filtration $\left\{\mathcal{F}_{t}\right\}$ is thus identified to be that generated by the information process $\left\{\xi_{t}\right\}_{0 \leq t \leq T}$. The price process of the asset that entails a single cash flow $X_{T}$ at time $T$, which in this case might be viewed as a credit-risky discount bond, is thus obtained according to the prescription:

$$
B_{t T}=P_{t T} \mathbb{E}^{\mathbb{Q}}\left[X_{T} \mid \mathcal{F}_{t}\right]
$$

Here we let $\left\{P_{t T}\right\}$ denotes the discount function, which for simplicity is assumed deterministic. We thus see that, once the pricing measure $\mathbb{Q}$ is fixed, there are two inputs; cash flow $X_{T}$ and market filtration $\left\{\mathcal{F}_{t}\right\}$, the specification of which leads to an output that is the price process $\left\{B_{t T}\right\}$.

The fact that it is market information that affects price dynamics has been emphasised by many authors (e.g., $[5,6]$ ), and has been demonstrated against market data more recently (e.g., [7-9]). The aim of the BHM approach is therefore to bring the mathematical abstraction of financial modelling at the level of the specification of market filtration. In this way, price process can be derived as an emergent phenomenon, rather than postulated from the outset. Motivated by this, here we analyse in detail properties of a specific model that corresponds to the simplest stochastic-volatility extension of the original BHM model introduced in [1].

One of the simplifying assumptions in the original BHM model (1) and the various generalisations of it that have appeared in the literature is that the information flow-rate parameter $\sigma$ is taken to be $\mathcal{F}_{0}$-measurable. In a more realistic setup, however, market participants have little knowledge about the value of $\sigma$. In fact, in most cases the information flow rate is not measurable even after the value of $X_{T}$ is revealed. The main issue addressed in this paper is therefore to extend the original BHM model to allow for $\sigma$ to be a random variable. Such an extension is shown to give rise to a sufficient flexibility to calibrate volatility surfaces of the original BHM model.

The paper is organised as follows. In $\S I I$ we begin by discussing the interpretation of the information process (1) as representing the totality of information available to the market concerning the value of $X_{T}$. In $\S$ III we work out a statistical measure of sensitivity of the BHM model against the choice of the parameter $\sigma$, thus indicating the region in the parameter space that is susceptive to the misspecification of $\sigma$ in the original BHM model. In $\S I V$ we show that there is a kind of complementarity relation that holds between the information flow rate $\sigma$ and the cash flow $X_{T}$, and that they cannot both take arbitrary values owing to the measurability condition. In $\S \mathrm{V}$ we derive the expression for the defaultable bond price process in the random $\sigma$ environment. The associated price dynamics is worked out in $\S \mathrm{VI}$. In $\S \mathrm{VII}$ and $\S \mathrm{VIII}$ we carry out a detailed numerical analysis to reveal a range of subtle details about the price dynamics resulting from the model. The pricing of an option on the defaultable bond is worked out in $\S I X$. We conclude in $\S \mathrm{X}$ with a sketch of an idea on how we can model price manipulation within the information-based framework.

\section{MEANING OF THE INFORMATION PROCESS}

Before we proceed to investigate the properties of the model with a random $\sigma$ in detail, let us first comment on the meaning of the information process (1). In general, in a financial 
market, even if we accept the simplifying assumption implicit in (1) that the only relevant market factor is the cash flow $X_{T}$, there are plenty of information sources available for $X_{T}$, each being obscured by noise. We can therefore represent each of the information source in the signal-plus-noise form (1) and write

$$
\left\{\begin{array}{c}
\xi_{t}^{1}=\sigma_{1} X_{T} t+\beta_{t T}^{1} \\
\vdots \\
\xi_{t}^{n}=\sigma_{n} X_{T} t+\beta_{t T}^{n}
\end{array}\right.
$$

for the family of information processes available in the market concerning the impending cash flow $X_{T}$. The various noise processes $\left\{\beta_{t T}^{i}\right\}_{i=1, \ldots, n}$ in general may be mutually correlated (with correlation matrix $\rho$ ), but they are all independent of $X_{T}$.

An important point to observe now is the fact that the aggregate information processes (3) is somewhat redundant; the information relevant to the cash flow $X_{T}$ contained in (3) can be represented in the form of a single information process (1), with the choice

$$
\sigma^{2}=\frac{\sum_{i}^{n} \sigma_{i}^{2} \rho_{i i}^{-1}-2 \Sigma_{i \neq j} \sigma_{i} \sigma_{j} \rho_{i j}^{-1}}{\operatorname{det}(\rho)}
$$

for the effective information flow rate, and

$$
\beta_{t T}=\frac{1}{\sigma}\left(\Sigma_{i, j}^{N} \sigma_{i} \rho_{i j}^{-1} \beta_{t T}^{i}\right)
$$

for the effective noise. Here $\rho_{i j}^{-1}$ denotes the $i j$ element of the inverse correlation matrix.

Put the matter differently, the filtration generated jointly by the set of information processes (3) is equivalent to the filtration generated jointly by the single information process (1) and a family of noise processes given by combinations of $\left\{\beta_{t T}^{i}\right\}$. However, since noise terms are independent of $X_{T}$, they make no contribution towards the pricing of that asset entailing the cash flow $X_{T}$. We can therefore discard them altogether and represent the totality of 'relevant' information in the form of a single information process (1).

Remark: In the case of a pair of information processes on the same market factor $X_{T}$, the construction of an effective information process is used in [10] to characterise the behaviour of an informed trader having access to additional noisy information.

\section{SENSITIVITY ANALYSIS}

It is of interest to identify the sensitivity of the BHM model (1) to the specification of the information flow-rate parameter $\sigma$, given the fact that the value of $\sigma$ is usually unknown. Often one considers the option vega as a measure of parameter sensitivity, but here we are interested in a global measure of parameter sensitivity. The result will be useful in identifying the region in the parameter space for which a misspecification of the flow-rate parameter $\sigma$ yields significant errors in pricing a range of products, not just vanilla options.

A universal measure of sensitivity in statistical analysis is given by the Fisher information [11]. To work out the Fisher information associated with the parameter $\sigma$ we proceed as follows. For simplicity, let as assume that the cash flow $X_{T}$ takes discrete values $\left\{x_{i}\right\}$ with a priori probability $\left\{p_{i}\right\}$. To determine the information measure of Fisher we need the 
expression for the a posteriori probability $\pi_{i t}=\mathbb{Q}\left(X_{T}=x_{i} \mid \mathcal{F}_{t}\right)$. It is shown in [1], by making use of the Bayes formula, that this is given by

$$
\pi_{i t}=\frac{p_{i} \exp \left(\frac{T}{T-t}\left(\sigma x_{i} \xi_{t}-\frac{1}{2} \sigma^{2} x_{i}^{2} t\right)\right)}{\sum p_{i} \exp \left(\frac{T}{T-t}\left(\sigma x_{i} \xi_{t}-\frac{1}{2} \sigma^{2} x_{i}^{2} t\right)\right)}
$$

We can therefore regard $\left\{\pi_{i t}\right\}=\left\{\pi_{i t}(\sigma)\right\}$ as a one-parameter family of probabilities. The Fisher information $g_{t}(\sigma)$ associated with the parameter $\sigma$ is then defined by the expression:

$$
g_{t}(\sigma)=\sum_{i} \frac{1}{\pi_{i t}(\sigma)}\left(\frac{\partial \pi_{i t}(\sigma)}{\partial \sigma}\right)^{2} .
$$

Remark: It is interesting that the Fisher information in the case of the BHM model (1) has the interpretation in terms of the conditional variance:

$$
g_{t}(\sigma)=\sigma t\left(\frac{T}{T-t}\right)^{2} \operatorname{var}\left(X_{T} \beta_{t T} \mid \xi_{t}\right)
$$

To see this, let us define

$$
p_{i t}(\sigma)=p_{i} \exp \left(\frac{T}{T-t}\left(\sigma x_{i} \xi_{t}-\frac{1}{2} \sigma^{2} x_{i}^{2} t\right)\right)
$$

so that $\pi_{i t}=p_{i t} / \sum_{i} p_{i t}$. We then have

$$
\frac{\partial p_{i t}(\sigma)}{\partial \sigma}=\frac{T}{T-t}\left(x_{i} \xi_{t}-\sigma x_{i}^{2} t\right) p_{i t}(\sigma)
$$

from which it follows that

$$
\begin{aligned}
\frac{\partial \pi_{i t}}{\partial \sigma} & =\frac{1}{\left(\sum_{i} p_{i t}\right)^{2}}\left[\frac{\partial p_{i t}}{\partial \sigma} \sum_{i} p_{i t}-p_{i t} \sum_{i} \frac{\partial p_{i t}}{\partial \sigma}\right] \\
& =\frac{T}{T-t} p_{i t}\left(\frac{x_{i} \xi_{t}-\sigma x_{i}^{2} t}{\sum_{i} p_{i t}}-\frac{\sum_{i} p_{i t}\left(x_{i} \xi_{t}-\sigma x_{i}^{2} t\right)}{\left(\sum_{i} p_{i t}\right)^{2}}\right) .
\end{aligned}
$$

Therefore, we obtain

$$
g_{t}(\sigma)=\left(\frac{T}{T-t}\right)^{2} \frac{\sum_{i} p_{i t}\left(x_{i} \xi_{t}-\sigma x_{i}^{2} t-\frac{\sum_{i} p_{i t}\left(x_{i} \xi_{t}-\sigma x_{i}^{2} t\right)}{\sum_{i} p_{i t}}\right)^{2}}{\sum p_{i t}}
$$

and by substitution of (1) in (12) we deduce (8).

The reason that $g_{t}(\sigma)$ measures the parameter sensitivity follows from the celebrated Cramér-Rao inequality, which shows that the variance of the parameter estimate is bounded below by the inverse of the Fisher information. Additionally, as observed by Rao [12], the separation, i.e. the divergence measure associated with two models characterised by $\pi_{i t}(\sigma)$ and $\pi_{i t}\left(\sigma^{\prime}\right)$ is determined by the integral:

$$
D_{t}\left(\sigma, \sigma^{\prime}\right)=\int_{\sigma}^{\sigma^{\prime}} \sqrt{g_{t}(u)} \mathrm{d} u
$$




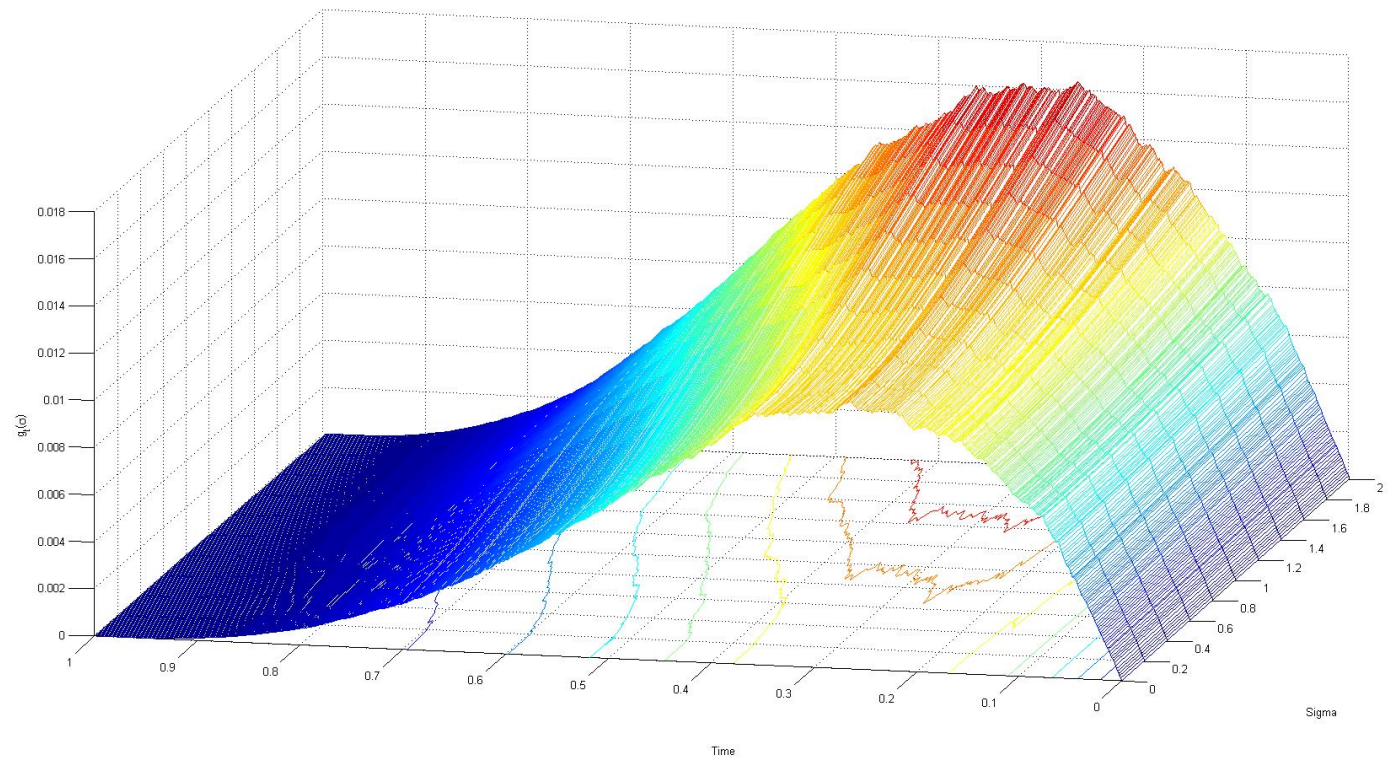

FIG. 1: The unconditional expectation $\mathbb{E}\left[g_{t}(\sigma)\right]$ of the Fisher information for different values of $t$. The numerical result is obtained by averaging over 1,000 sample paths. The parameters are chosen such that $\left\{x_{i}\right\}=\{0,0.5,1\},\left\{p_{i}\right\}=\{0.1,0.15,0.75\}$, and $T=1$. Where the value of $\mathbb{E}\left[g_{t}(\sigma)\right]$ is high, the model is on average sensitive to the choice of the specification of the parameter $\sigma$.

In figure 1 we show the numerical plot of the expectation $\mathbb{E}\left[g_{t}(\sigma)\right]$ of the Fisher information for different values of $t$, averaged over 1,000 sample paths. The parameters are chosen such that $\left\{x_{i}\right\}=\{0,0.5,1\},\left\{p_{i}\right\}=\{0.1,0.15,0.75\}$, and $T=1$. We observe that the Fisher information is increasing in $\sigma$. It is evident from the plot that the basic BHM model (1) is sensitive to the choice of the information flow rate parameter $\sigma$ up until about two thirds of the way into the duration of the contract. The price of a generic derivative on a credit risky bond, with maturity not too close to zero but also shorter than two-third of the bond maturity, is thus likely to be sensitive to the specification of the parameter $\sigma$ in this model. This sensitivity can be made more robust by allowing $\sigma$ to be random.

\section{QUANTISATION OF INFORMATION FLOW RATE}

As indicated above, the complicated way in which the market information flow rate $\sigma$ depends on individual information flow rates $\left\{\sigma_{i}\right\}$, as represented in (4), suggests that it is not reasonable to assume that market participants have access to the value of $\sigma$. Indeed, in many realistic setup it is unlikely that market participants will ever learn the value of $\sigma$, even after the value of $X_{T}$ is revealed (cf. [9]). More generally, the value of $\sigma$ can change over time in a random manner. Here we will be considering the simplest such situation in which $\sigma$ is given by a fixed random variable independent of $X_{T}$ and $\left\{\beta_{t T}\right\}$. Before we proceed, however, we draw attention to the fact that whether $\sigma$ is a fixed random variable or a more-general stochastic process, the $\mathcal{F}_{T}$-measurability of $X_{T}$ enforces a constraint on the allowable choice for $\sigma$.

To see this, let us consider a simple example. Suppose that the random cash flow $X_{T}$ 
takes three possible values $\{0,0.5,1\}$ with a priori probability $\left\{p_{1}, p_{2}, p_{3}\right\}$, and $\sigma$ takes two possible values $\{0.5,1\}$ with a priori probability $\left\{q_{1}, q_{2}\right\}$. Then there are four possible realised values for the terminal information $\xi_{T}$ :

$$
\xi_{T}=\sigma T X_{T}= \begin{cases}0 & \Rightarrow X_{T}=0, \quad \sigma=0.5 \text { or } 1 \\ 0.25 T & \Rightarrow\left(X_{T}, \sigma\right)=(0.5,0.5) \\ 0.5 T & \Rightarrow\left(X_{T}, \sigma\right)=(0.5,1) \text { or }(1,0.5) \\ T & \Rightarrow\left(X_{T}, \sigma\right)=(1,1)\end{cases}
$$

In this example, if the realised value of $\xi_{T}$ happens to be $0,0.25 T$, or $T$, then we can unambiguously determine the value of $X_{T}$ irrespective of what the value of $\sigma$ might have been; whereas if the outcome happens to be $0.5 T$, then the value of $X_{T}$ could be 0.5 or 1 .

This example illustrates the fact that once the cash flow random variable is modelled, the information flow rate cannot take an arbitrary random variable. In particular, $\sigma$ can not be a continuous random variable. (More generally, if $\sigma=\sigma(t)$ is time dependent, then $\sigma(t)$ for $t<T$ can be arbitrary, but the constraint discussed here remains applicable for the terminal value $\sigma(T)$.) Therefore, there is a kind of 'quantisation' condition imposed on $\sigma$. For the same token, in the random- $\sigma$ environment, the cash flow $X_{T}$ cannot be a continuous variable - this does not pose real constraints because cash flows are strictly speaking never continuous in reality.

\section{PRICE PROCESS WITH A RANDOM INFORMATION FLOW RATE}

Bearing in mind the quantisation condition imposed by the measurability of $X_{T}$, we proceed to consider the random- $\sigma$ extension of the BHM model. Specifically, we let $X_{T}$ take the values $\left\{x_{i}\right\}_{i=1, \ldots, n}$ with a priori probabilities $\left\{p_{i}\right\}_{i=1, \ldots, n}$; and let $\sigma$ take the values $\left\{\sigma_{k}\right\}_{k=1, \ldots, m}$ with a priori probabilities $\left\{q_{k}\right\}_{k=1, \ldots, m}$. These random variables are chosen such that degenerate situations like the example given in (14) are excluded, and hence $X_{T}$ is ensured to be $\mathcal{F}_{T}$-measurable.

To determine the conditional expectation $\mathbb{E}\left[X_{T} \mid \mathcal{F}_{t}\right]$ we determine first the conditional probability $\pi_{i t}=\mathbb{Q}\left(X_{T}=x_{i} \mid \mathcal{F}_{t}\right)$. A calculation making use of the Bayes formula shows that this is given by

$$
\pi_{i t}=\frac{\sum_{k=1}^{m} p_{i} q_{k} \exp \left[\frac{T}{T-t}\left(x_{i} \sigma_{k} \xi_{t}-\frac{1}{2} x_{i}^{2} \sigma_{k}^{2} t\right)\right]}{\sum_{s=1}^{n} \sum_{l=1}^{m} p_{s} q_{l} \exp \left[\frac{T}{T-t}\left(x_{s} \sigma_{l} \xi_{t}-\frac{1}{2} x_{s}^{2} \sigma_{l}^{2} t\right)\right]} .
$$

It follows that the bond price is give by:

$$
B_{t T}=P_{t T} \frac{\sum_{i}^{n} \sum_{k=1}^{m} x_{i} p_{i} q_{k} \exp \left[\frac{T}{T-t}\left(x_{i} \sigma_{k} \xi_{t}-\frac{1}{2} x_{i}^{2} \sigma_{k}^{2} t\right)\right]}{\sum_{s=1}^{n} \sum_{l=1}^{m} p_{s} q_{l} \exp \left[\frac{T}{T-t}\left(x_{s} \sigma_{l} \xi_{t}-\frac{1}{2} x_{s}^{2} \sigma_{l}^{2} t\right)\right]} .
$$

The derivation of (15) follows closely that of the original BHM model in [1]. First, we show that the information process (1) with a random $\sigma$ is a Markov process satisfying

$$
\mathbb{Q}\left(\xi_{t} \leq x \mid \mathcal{F}_{s}\right)=\mathbb{Q}\left(\xi_{t} \leq x \mid \xi_{s}\right)
$$

for all $x \in \mathbb{R}$ and all $s, t$ such that $0 \leq s \leq t \leq T$. To establish this, it suffices to show that

$$
\mathbb{Q}\left(\xi_{t} \leq x \mid \xi_{s}, \xi_{s_{1}}, \ldots, \xi_{s_{k}}\right)=\mathbb{Q}\left(\xi_{t} \leq x \mid \xi_{s}\right)
$$


for any collection of times $t, s, s_{1}, \ldots, s_{k}$ such that $T \geq t>s>s_{1}>\ldots>s_{k}>0$. We recall first that from properties of a Brownian bridge process it follows that $\left(\beta_{s T} / s-\beta_{s_{1} T} / s_{1}\right)$ and $\left(\beta_{s_{2} T} / s_{2}-\beta_{s_{3} T} / s_{3}\right)$ are independent. We now observe that

$$
\begin{aligned}
\mathbb{Q}\left(\xi_{t} \leq x \mid \xi_{s}, \xi_{s_{1}}, \cdots, \xi_{s_{k}}\right) & =\mathbb{Q}\left(\xi_{t} \leq x \mid \xi_{s}, \frac{\xi_{s}}{s}-\frac{\xi_{s_{1}}}{s_{1}}, \cdots, \frac{\xi_{s_{k-1}}}{s_{k-1}}-\frac{\xi_{s_{k}}}{s_{k}}\right) \\
& =\mathbb{Q}\left(\xi_{t} \leq x \mid \xi_{s}, \frac{\beta_{s T}}{s}-\frac{\beta_{s_{1} T}}{s_{1}}, \cdots, \frac{\beta_{s_{k-1} T}}{s_{k-1}}-\frac{\beta_{s_{k} T}}{s_{k}}\right),
\end{aligned}
$$

but since $\xi_{s}$ and $\xi_{t}$ are independent of the remaining variables $\beta_{s T} / s-\beta_{s_{1} T} / s_{1}, \cdots$, $\beta_{s_{k-1} T} / s_{k-1}-\beta_{s_{k} T} / s_{k}$, the desired Markov property follows.

From the Markovian property of $\left\{\xi_{t}\right\}$ the problem of determining the conditional probability process $\left\{\pi_{i t}\right\}$ simplifies to calculating the conditional probability $\mathbb{Q}\left(X_{T}=x_{i} \mid \xi_{t}\right)$. Then from the Bayes formula we find

$$
\mathbb{Q}\left(X_{T}=x_{i} \mid \xi_{t}\right)=\frac{\mathbb{Q}\left(X_{T}=x_{i}\right) \rho\left(\xi_{t} \mid X_{T}=x_{i}\right)}{\sum_{i} \mathbb{Q}\left(X_{T}=x_{i}\right) \rho\left(\xi_{t} \mid X_{T}=x_{i}\right)},
$$

where $\rho\left(\xi \mid X_{T}=x_{i}\right)$ is the conditional density for $\xi_{t}$. But from

$$
\rho\left(\xi_{t} \mid X_{T}=x_{i}\right)=\sum_{k} \rho\left(\xi_{t} \mid X_{T}=x_{i}, \sigma=\sigma_{k}\right) \mathbb{Q}\left(\sigma=\sigma_{k}\right)
$$

we deduce that

$$
\pi_{i t}=\frac{\sum_{k} p_{i} q_{k} \rho\left(\xi_{t} \mid X_{T}=x_{i}, \sigma=\sigma_{k}\right)}{\sum_{i} \sum_{k} p_{i} q_{k} \rho\left(\xi_{t} \mid X_{T}=x_{i}, \sigma=\sigma_{k}\right)} .
$$

Conditional on $X_{T}=x_{i}$ and $\sigma=\sigma_{k}$ the random variable $\xi_{t}=\sigma X_{T} t+\beta_{t T}$ has the probability law of a drifted Brownian bridge:

$$
\rho\left(\xi_{t} \mid X_{T}=x_{i}, \sigma=\sigma_{k}\right)=\frac{1}{\sqrt{2 \pi t(T-t) / T}} \exp \left(-\frac{1}{2} \frac{\left(\xi_{t}-\sigma_{k} x_{i} t\right)^{2}}{t(T-t) / T}\right) .
$$

Substituting (23) in (22) we deduce the desired expression (15).

\section{PRICE DYNAMICS}

With the expression (16) for the price process at hand we are able to investigate its dynamics. To proceed let us write $p_{i k t}=p_{i k}\left(t, \xi_{t}\right)$, where

$$
p_{i k}(t, \xi)=p_{i} q_{k} \exp \left[\frac{T}{T-t}\left(x_{i} \sigma_{k} \xi-\frac{1}{2} x_{i}^{2} \sigma_{k}^{2} t\right)\right] .
$$

Then an application of Ito's lemma gives

$$
\frac{\mathrm{d} p_{i k t}}{p_{i k t}}=\frac{\sigma_{k} T}{T-t} x_{i}\left(\mathrm{~d} \xi_{t}+\frac{\xi_{t}}{T-t} \mathrm{~d} t\right) .
$$


It follows that the process $\left\{p_{i t}\right\}$ defined by

$$
p_{i t}=\sum_{k} p_{i k t}
$$

fulfils the stochastic equation

$$
\mathrm{d} p_{i t}=\frac{T}{T-t} x_{i}\left(\sum_{k} \sigma_{k} p_{i k t}\right)\left(\mathrm{d} \xi_{t}+\frac{\xi_{t}}{T-t} \mathrm{~d} t\right) .
$$

Since the a posteriori density is given by

$$
\pi_{i t}=\frac{p_{i t}}{\sum_{i} p_{i t}}
$$

another application of Ito's rule gives

$$
\mathrm{d} \pi_{i t}=\frac{T}{T-t}\left(x_{i} \frac{\sum_{k} \sigma_{k} p_{i k t}}{\sum_{i} p_{i t}}-\pi_{i t} \mathbb{E}\left[\sigma X_{T} \mid \xi_{t}\right]\right) \mathrm{d} W_{t}
$$

where

$$
\mathrm{d} W_{t}=\mathrm{d} \xi_{t}+\frac{1}{T-t}\left(\xi_{t}-T \mathbb{E}\left[\sigma X_{T} \mid \xi_{t}\right]\right) \mathrm{d} t .
$$

Putting these together, we find that the dynamical equation satisfied by the defaultable discount bond price is given by

$$
\mathrm{d} B_{t T}=r_{t} B_{t T} \mathrm{~d} t+\Sigma_{t T} \mathrm{~d} W_{t}
$$

where the volatility process is determined by the conditional covariance process of $X_{T}$ and $\sigma X_{T}$ :

$$
\Sigma_{t T}=P_{t T} \frac{T}{T-t} \operatorname{cov}\left(X_{T}, \sigma X_{T} \mid \xi_{t}\right)
$$

In particular, if $\sigma$ is constant, the covariance reduces to the variance of $X_{T}$, and we recover the original BHM model. We remark that although $\sigma$ and $X_{T}$ are a priori independent, they are not conditionally independent, and hence the covariance term does not reduce to a simpler expression $\mathbb{E}\left[\sigma \mid \xi_{t}\right] \operatorname{var}\left(X_{T} \mid \xi_{t}\right)$.

Next, we establish that the process $\left\{W_{t}\right\}$ defined in (30) is the innovations representation associated with the filtering problem corresponding to the information process $\left\{\xi_{t}\right\}$. We shall follow closely the argument of [1] but extended to a random $\sigma$. Since $\left(\mathrm{d} W_{t}\right)^{2}=\mathrm{d} t$, to show that $\left\{W_{t}\right\}$ is a Brownian motion it suffices to establish that it is a martingale. For $t \leq u$ we have

$$
\begin{aligned}
\mathbb{E}\left[W_{u} \mid \mathcal{F}_{t}\right]= & W_{t}+\mathbb{E}\left[\left(\xi_{u}-\xi_{t}\right) \mid \xi_{t}\right]+\mathbb{E}\left[\int_{t}^{u} \frac{\xi_{s}}{T-s} \mathrm{~d} s \mid \xi_{t}\right]-T \mathbb{E}\left[\int_{t}^{u} \frac{\mathbb{E}\left[\sigma X_{T} \mid \xi_{s}\right]}{T-s} \mathrm{~d} s \mid \xi_{t}\right] \\
= & W_{t}+\mathbb{E}\left[\sigma X_{T} u+\beta_{u T} \mid \xi_{t}\right]-\mathbb{E}\left[\sigma X_{T} t+\beta_{t T} \mid \xi_{t}\right]+\mathbb{E}\left[\sigma X_{T} \mid \xi_{t}\right] \int_{t}^{u} \frac{s}{T-s} \mathrm{~d} s \\
& +\mathbb{E}\left[\int_{t}^{u} \frac{\beta_{s T}}{T-s} \mathrm{~d} s \mid \xi_{t}\right]-\mathbb{E}\left[\sigma X_{T} \mid \xi_{t}\right] \int_{t}^{u} \frac{T}{T-s} \mathrm{~d} s .
\end{aligned}
$$


Evidently, the coefficients of $\mathbb{E}\left[\sigma X_{T} \mid \xi_{t}\right]$ cancel, and we are left with

$$
\mathbb{E}\left[W_{u} \mid \mathcal{F}_{t}\right]=W_{t}+\mathbb{E}\left[\beta_{u T} \mid \xi_{t}\right]-\mathbb{E}\left[\beta_{t T} \mid \xi_{t}\right]+\int_{t}^{u} \frac{1}{T-s} \mathbb{E}\left[\beta_{s T} \mid \xi_{t}\right] \mathrm{d} s
$$

but because

$$
\mathbb{E}\left[\beta_{u T} \mid \xi_{t}\right]=\frac{T-u}{T-t} \mathbb{E}\left[\beta_{t T} \mid \xi_{t}\right]
$$

we deduce the martingale condition

$$
\mathbb{E}\left[W_{u} \mid \mathcal{F}_{t}\right]=W_{t}
$$

It follows that the process $\left\{W_{t}\right\}$ defined via (30) is indeed a $\mathbb{Q}$-Brownian motion with respect to the filtration generated by the information process $\left\{\xi_{t}\right\}$.

\section{NUMERICAL ANALYSIS OF THE SAMPLE-PATH BEHAVIOUR}

In this section we analyse the sample-path behaviour of a defaultable digital bond price under our uncertain information model through simulation studies. This provides us with a better intuitive understanding of the characteristics of the model under study.

In figure 2 and 3 we have shown simulations of sample paths of the bond price processes, the corresponding averaged (over five sample paths) volatility process, and the averaged (again, over five sample paths) vol-of-vol process. In these plots, we have set $r=0 \%$ for simplicity; maturity of the digital bond is $T=1$. The information flow rate in figure 2 is chosen to be a binary random variable taking values $\{0.6,0.8\}$ with an equal a priori probability; whereas in figure $3, \sigma$ takes the values $\{0.4,1.0\}$ also with an equal a priori probability. Hence in both cases we have $\mathbb{E}[\sigma]=0.7$, but they have different standard deviations. The simulation study shows that as we increase the variance of $\sigma$ the variance of the price paths increases. This behaviour is intuitively expected although not immediately apparent from the formula for the bond volatility.

One other interesting observation to be drawn from the simulation studies, as compared to the original BHM model, is that the degree of variation of the sample paths, or simply the volatility, is smaller than those observed in the BHM with the same $\sigma$ value. For example, although one of the sample paths in figure 3 has the value $\sigma=1$, which in the BHM model would have caused the paths to reach its terminal value at about four-fifth of the way, such a large variation is not present in our uncertain information model. This is because the conditional expectation of $X_{T}$ involves products of the a priori probability $\left\{q_{k}\right\}$, and this 'dampens' the overall variability. If we set one of the $q_{k}$ 's equal to zero, which reduces the model to the original BHM, then the damping effect disappears. This behaviour is plausible because market participants are uncertain about the true information flow rate; their knowledge of the information is made additionally fuzzy by the uncertainty in $\sigma$, and hence it generally takes a longer time to discover the true terminal cash flow. This uncertainty will, consequently, and perhaps at first surprisingly, reduce the overall volatility for the price process. The reduction in volatility becomes apparent when we look at the average of the processes.

The volatility of the processes in some sense reflects the 'learning rate' of the terminal value of the bond price (or, equivalently, the value of $X_{T}$ ). This learning rate is somewhat 


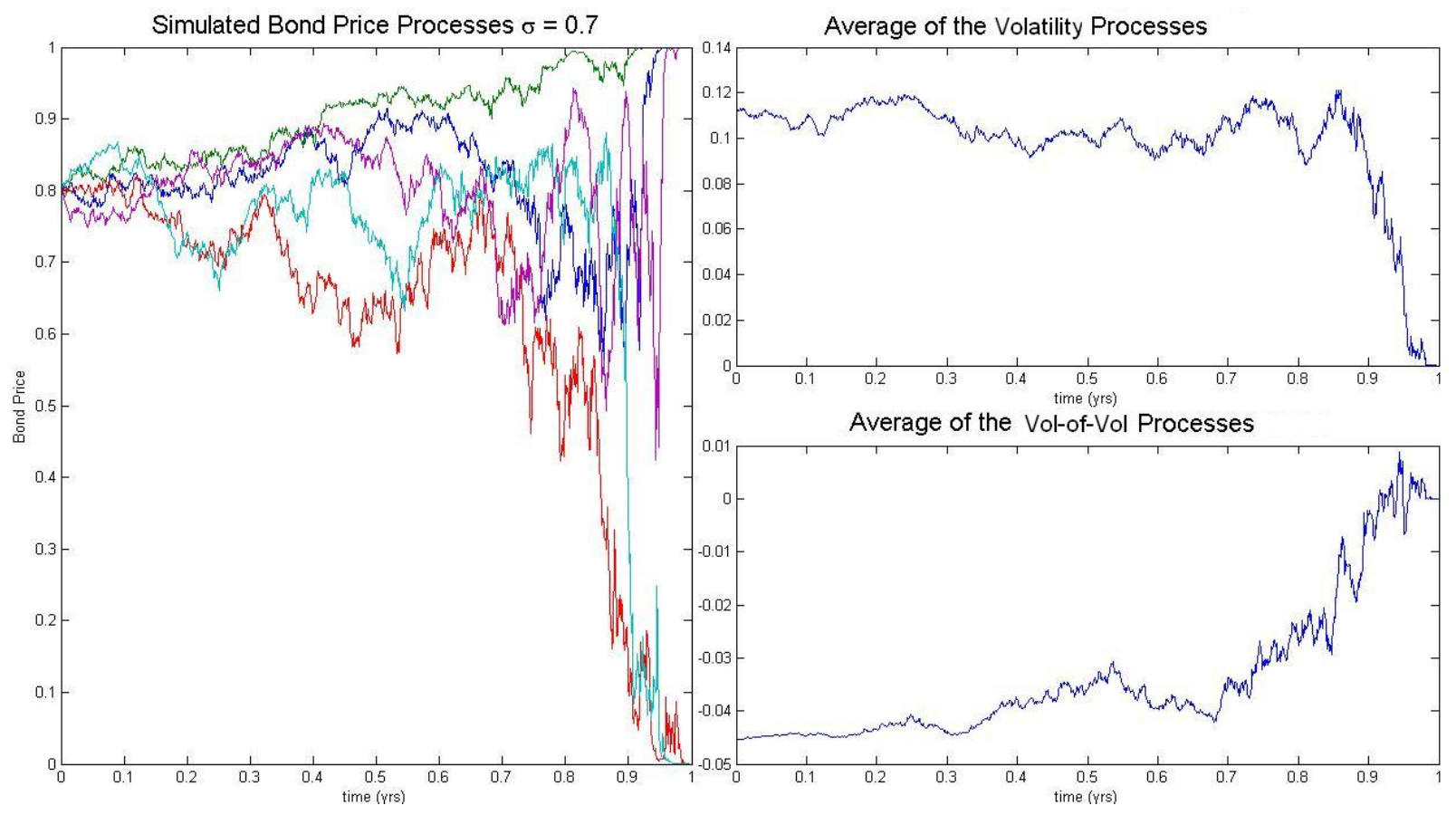

FIG. 2: Simulated Bond price (left), volatility (top right) and Vol-of-Vol (bottom right) processes in uncertain information model with $\sigma=\{0.6,0.8\}, q_{k}=\{0.5,0.5\}, X_{T}=\{0,1\}, p_{i}=\{0.2,0.8\}$, $r=0 \%$ and $T=1$.

slowed down in the uncertain information model, hence resulting in the dampening of the volatility. If the standard deviation of $\sigma$ is very small, then the volatility process almost matches that of the BHM model. However, as the standard deviation of $\sigma$ is made wider so that the true information becomes less clear, this leads to the reduction in the price volatility. Nevertheless, as time passes, the value of $X_{T}$ must eventually be revealed, and this leads to a subtle behaviour in the averaged volatility.

In view of this, we investigate further the behaviour of the volatility processes. Because in our example here the random variable $\sigma$ is chosen such that it is not measurable for all $t \in[0, T]$, we can expect some degree of uncertainty to sustain until the very last moment. Indeed, the behaviour of the price process in the uncertain information model is not entirely counterintuitive; we expect, for example, that the price process behaves like a mixture of the BHM with the probabilities $\left\{q_{k}\right\}$ denoting the weights of the possible scenarios of $\sigma_{k}$. That this intuition is more or less correct, albeit there are subtle details, is illustrated in figure 4, in which the mean volatility $\bar{\Sigma}_{t T}=\mathbb{E}\left[\Sigma_{t T}\right]$ associated with various distributions for $\sigma$ are compared. The two solid lines represent $\bar{\Sigma}_{t T}$ corresponding to the original BHM model, with the top line at $t=0$ taking the value $\sigma=0.9$ and bottom line taking the value $\sigma=0.5$. The dashed lines are mean volatilities generated by our uncertain information model. They take values $\sigma_{k}=\{0.5,0.9\}$ with the following probabilities: from the top at $t=0, q_{k}=\{0,1\}$; $q_{k}=\{0.1,0.9\} ; q_{k}=\{0.2,0.8\} ; q_{k}=\{0.3,0.7\}$, and so on, until $q_{k}=\{1,0\}$ for the bottom dashed line that coincides with the solid line.

The result reveals the following features: First, we see that when $\sigma=0.5$ the average volatility starts with a lower value as compared to that of $\sigma=0.9$; but eventually overrides the latter because information is revealed towards the end, and hence causing higher volatility. Second, we see that as we shift the mean of $\sigma$ from 0.9 to 0.5 , the mean volatility shifts 


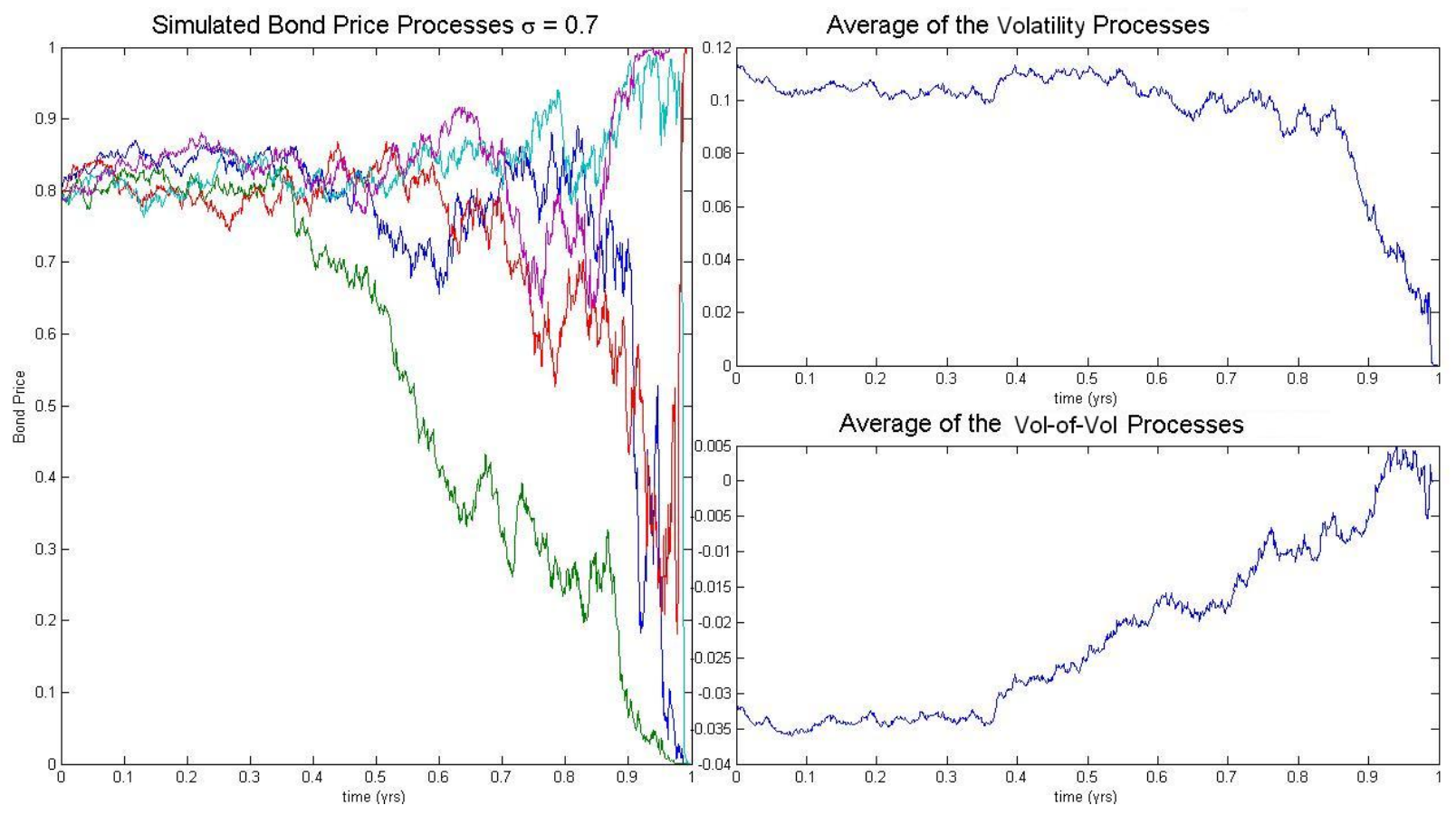

FIG. 3: Simulated Bond price (left), volatility (top right) and Vol-of-Vol (bottom right) processes in uncertain information model with $\sigma=\{0.4,1\}, q_{k}=\{0.5,0.5\}, X_{T}=\{0,1\}, p_{i}=\{0.2,0.8\}$, $r=0 \%$ and $T=1$.

accordingly. The graph shows that the mean volatility of the random $\sigma$ model behaves like a weighted sum of the BHM mean volatilities, with weights given by the a priori probability $\left\{q_{k}\right\}$. In this regard, the random information model studied here can be viewed as a BHM mixture model.

We also draw attention to the limiting behaviour of the mean volatility as $t \rightarrow T$. In figure 5 we see clearly that the mean volatility of the random information model does not reach zero when $t$ is very close to $T$, even though that of the BHM model has. This indicates that there are 'last minute surprises' because information regarding the terminal value is still uncertain, due to the additional uncertainty in $\sigma$. This is the essence of the random information flow rate model, as it describes the uncertainty in this market up until the last moment. Note, however, that values of mean volatility above $t \gtrsim 0.996 T$ were not available numerically, due to the appearance of large numbers.

\section{MUTUAL INFORMATION ANALYSIS}

An alternative way of investigating properties of the price process is to study the behaviour of mutual information between $B_{t T}$ and $X_{T}$, or, equivalently, between $\xi_{t}$ and $X_{T}$. This is the quantity that measures the amount of information contained in the asset price about the value of the impending cash flow (cf. [13, 14]), and thus represents how much the market has learned about the value of $X_{T}$ [10]. The mutual information is obtained by 


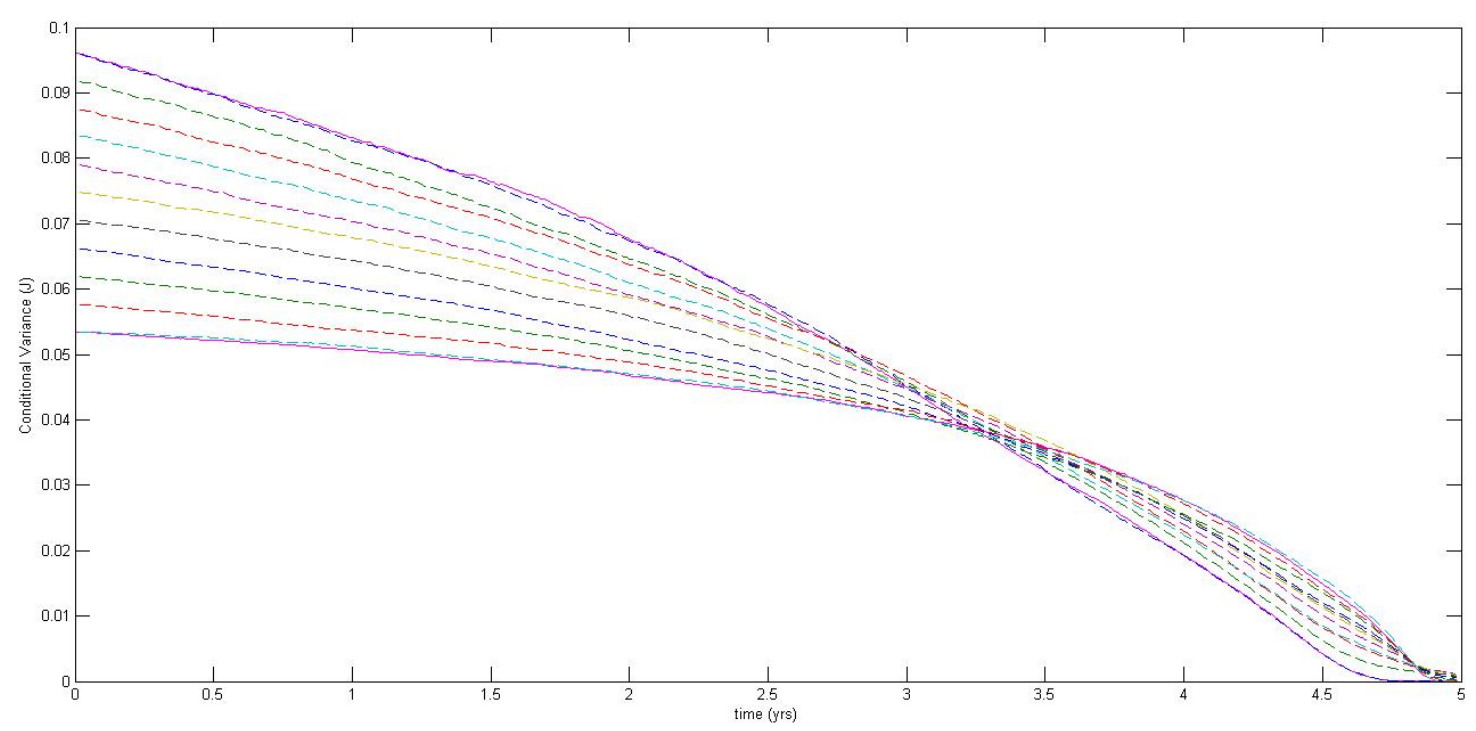

FIG. 4: Each line represents the average of 5,000 paths of volatility processes. The solid lines are that of the original BHM model with the top and bottom line taking $\sigma=0.9$ and $\sigma=0.5$ respectively, $X_{T}=\{0,1\}, p_{i}=\{0.2,0.8\}, r=0 \%$ and $T=5$. The dashed lines are mean volatilities of the uncertain information model taking values, from top to bottom respectively, $\sigma_{k}=\{0.5,0.9\}$ with $q_{k}=\{0,1\}, q_{k}=\{0.1,0.9\}, q_{k}=\{0.2,0.8\}, \ldots$ and $q_{k}=\{1,0\}$.

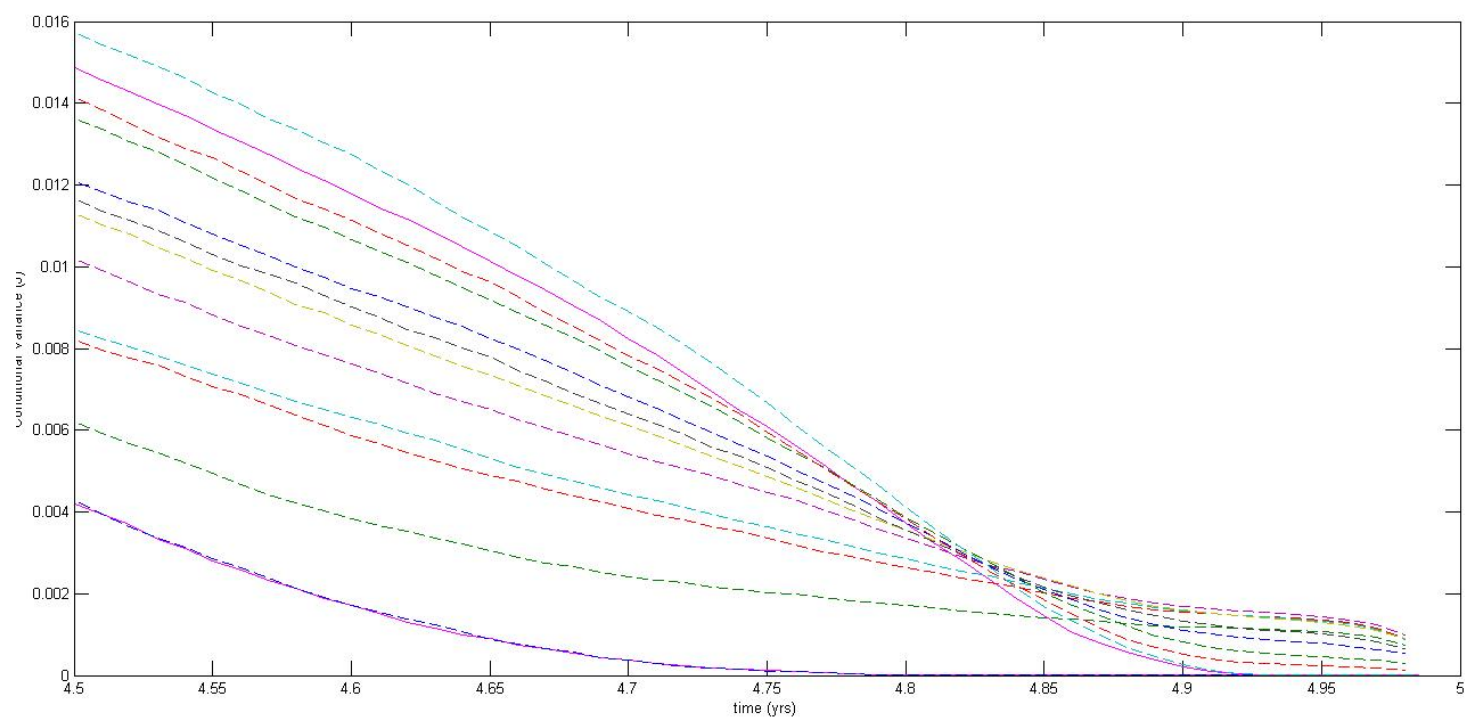

FIG. 5: Magnification of the averages of volatility processes. Each line represents the average of 5,000 paths of volatility processes. The solid lines are that of the original BHM model with the top and bottom line taking $\sigma=0.9$ and $\sigma=0.5$, respectively, $X_{T}=\{0,1\}, p_{i}=\{0.2,0.8\}, r=0 \%$ and $T=5$. The dashed lines are mean volatilities of the uncertain information model taking values, from top to bottom respectively, $\sigma_{k}=\{0.5,0.9\}$ with $q_{k}=\{0,1\}, q_{k}=\{0.1,0.9\}, q_{k}=\{0.2,0.8\}, \ldots$ and $q_{k}=\{1,0\}$. 
determining the expression:

$$
J\left(\xi_{t}, X_{T}\right)=\sum_{i}^{n} \int_{-\infty}^{\infty} \rho_{\xi X}(x, i) \ln \left(\frac{\rho_{\xi X}(x, i)}{\rho_{\xi}(x) \rho_{X}(i)}\right) \mathrm{d} x
$$

where

$$
\rho_{\xi X}(x, i)=\frac{\mathrm{d}}{\mathrm{d} x} \mathbb{Q}\left[\left(\xi_{t}<x\right) \cap\left(X_{T}=x_{i}\right)\right]
$$

is the joint density function of the random variables $\left(\xi_{t}, X_{T}\right)$, and $\rho_{\xi}, \rho_{X}$ are the respective marginal probabilities. By independence of $X_{T}$ and $\sigma$, we find that

$$
\mathbb{Q}\left[\left(\xi_{t}<x\right) \cap\left(X_{T}=x_{i}\right)\right]=\sum_{k}^{m} \mathbb{Q}\left(\xi_{t}<x \mid X_{T}=x_{i}, \sigma=\sigma_{k}\right) \mathbb{Q}\left(X_{T}=x_{i}\right) \mathbb{Q}\left(\sigma=\sigma_{k}\right),
$$

from which it follows that

$$
\rho_{\xi X}(x, i)=\sum_{k}^{m} q_{k} p_{i} \frac{1}{\sqrt{2 \pi t(T-t) / T}} \exp \left(-\frac{1}{2} \frac{\left(x-\sigma_{k} x_{i} t\right)^{2}}{t(T-t) / T}\right)
$$

since conditional on $X_{T}=x_{i}$ and $\sigma=\sigma_{k}$, the random variable $\xi_{t}$ is normally distributed with mean $\sigma_{k} x_{i} t$ and variance $t(T-t) / T$.

An alternative way of deriving the mutual information is via the formula

$$
J\left(\xi_{t}, X_{T}\right)=H_{0}-\mathbb{E}\left[H_{t}\right],
$$

where the Shannon-Wiener entropy $\left\{H_{t}\right\}$ is defined by the expression:

$$
H_{t}=-\sum_{i=1}^{n} \pi_{i t} \ln \pi_{i t}
$$

The entropy process $\left\{H_{t}\right\}_{0 \leq t<T}$ has the property that $\lim _{t \rightarrow T} H_{t}=0$. This follows from the fact that the conditional probability process $\left\{\pi_{i t}\right\}_{0 \leq t<T}$ has the limiting behaviour

$$
\lim _{t \rightarrow T} \pi_{i t}(\omega)=\mathbb{1}\left\{X_{T}(\omega)=x_{i}\right\}
$$

for $i=1, \ldots, n$. To see this, suppose that for a choice of $\omega \in \Omega$ we have $X_{T}(\omega)=x_{a}$ and $\sigma(\omega)=\sigma_{b}$ for some $a, b$. Conditional on this realisation the information process is given by $\xi_{t}=\sigma_{b} t x_{a}+\beta_{t T}$. Substituting this expression for $\xi_{t}$ into the expression for $\pi_{i t}$, and dividing the denominator and the numerator by the exponential factor containing $x_{a}$ and $\sigma_{b}$, we deduce that

$$
\pi_{a t}=\frac{p_{a}\left(q_{b}+\sum_{k \neq b} q_{k} \exp \left[\frac{T}{T-t}\left(x_{a}\left(\sigma_{k}-\sigma_{b}\right) \beta_{t T}-\frac{1}{2} x_{a}^{2}\left(\sigma_{k}-\sigma_{b}\right)^{2} t\right)\right]\right)}{p_{a} q_{b}+\sum_{i \neq a} \sum_{k \neq b} p_{i} q_{k} \exp \left[\frac{T}{T-t}\left(\left(x_{i} \sigma_{k}-x_{a} \sigma_{b}\right) \beta_{t T}-\frac{1}{2}\left(x_{i}^{2} \sigma_{j}^{2}-x_{a}^{2} \sigma_{b}^{2}\right) t\right)\right]} .
$$

As $t \rightarrow T$ all of the terms in the sums vanish. Therefore, $\lim _{t \rightarrow T} \pi_{a t}=1$ and furthermore, since $\sum_{i} \pi_{i t}=1$ for all $t$, we must have $\lim _{t \rightarrow T} \pi_{i t}=0$ for $i \neq a$. Finally, since

$$
H_{t}=-\ln \prod_{i=1}^{n} \pi_{i t}^{\pi_{i t}}
$$


we deduce that $\lim _{t \rightarrow T} H_{t}=0$.

In figure 6 we have a graphical illustration of the behaviour of mutual information. The idea here is to relate this with the average volatility that we observed in the previous section. We have used exactly the same parameter range to make the comparison more transparent. Hence, the mutual information for the BHM model with $\sigma=0.9$ and $\sigma=0.5$ form the upper and lower bounds, respectively, at early times in figure 6 . The mutual information curves that lie within these bounds at early times are those under the uncertain information model, taking the values $\sigma_{k}=\{0.5,0.9\}$ with probabilities $q_{k}=\{0,1\}, q_{k}=\{0.1,0.9\}, \cdots$, $q_{k}=\{1,0\}$, respectively, from top to bottom.

The results shown in the figure match exactly the findings from the mean volatility analysis. The gap in the plots close to $t \approx T$ is again due to numerical limitations. Observe the crossover pattern seen here, as we change the distribution of $\sigma$. The processes with a low mutual information at early times cross the higher ones at later stages because the volatility is greater towards the end so as to 'catch up' with the learning rate. Further, there are still uncertainties left until the very last moment.

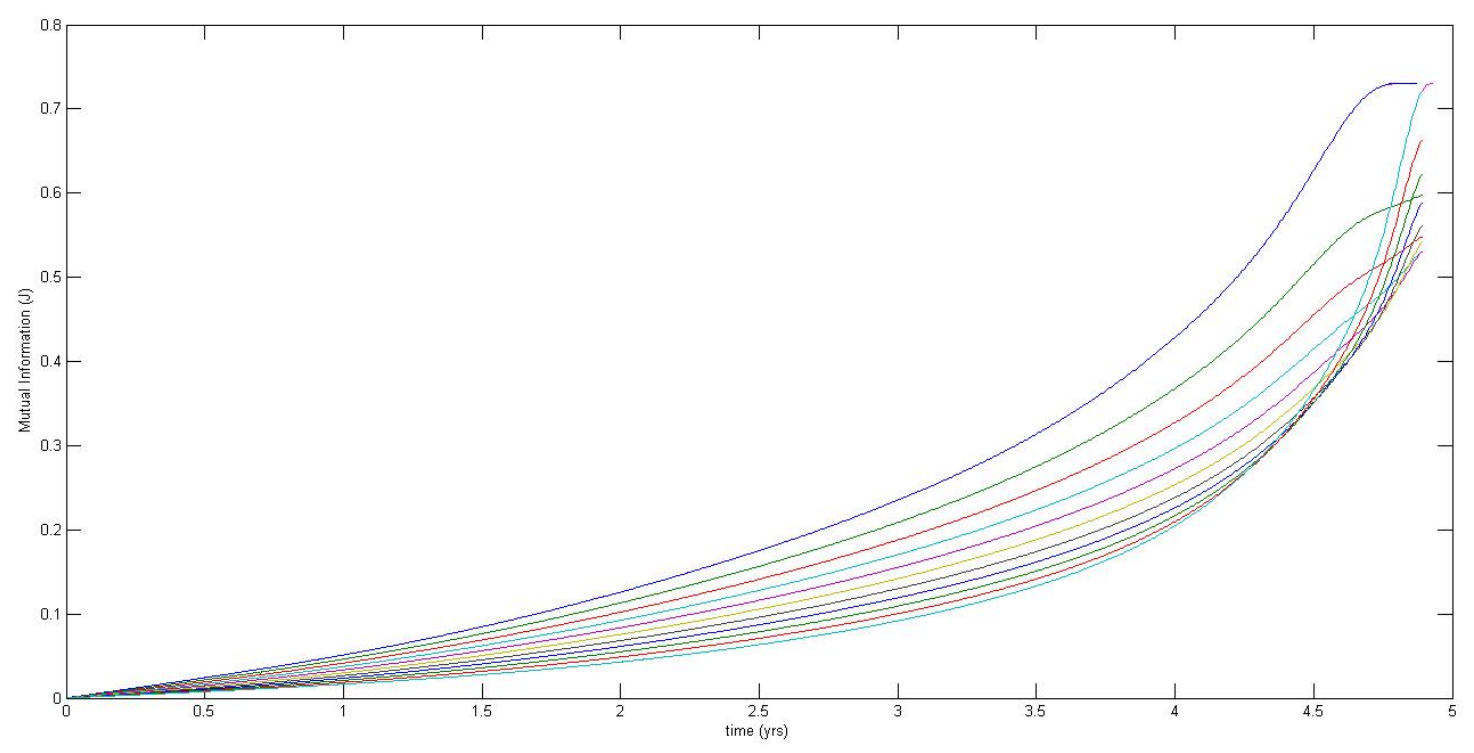

FIG. 6: Mutual information under the random $\sigma$ model. We let $\sigma_{k}=\{0.5,0.9\}$ with probabilities, from top to bottom at early times, $q_{k}=\{0,1\}, q_{k}=\{0.1,0.9\}, \ldots, q_{k}=\{1,0\}$ respectively; $X_{T}=$ $\{0,1\}, p_{i}=\{0.2,0.8\}$, and $T=5$.

\section{OPTION ON CREDIT-RISKY BONDS}

We now turn to the problem of pricing options on a credit-risky bond in our uncertain information flow-rate extension of the BHM model. Our analysis follows closely that given in [1]. Consider the valuation of a European-style call option on the defaultable discount bond maturing at $T$. The option maturity is $t \leq T$, and the strike is $K$. The initial value 
of the call option is:

$$
\begin{aligned}
C_{0} & =P_{0 t} \mathbb{E}\left[\left(B_{t T}-K\right)^{+}\right] \\
& =P_{0 t} \mathbb{E}\left[\left(\sum_{i} P_{t T} \pi_{i t} x_{i}-K\right)^{+}\right],
\end{aligned}
$$

where $\pi_{i t}$ is as given in (28). If we define the positive process $\left\{\Phi_{t}\right\}$ according to

$$
\Phi_{t}=\sum_{i} \sum_{k} p_{i k t}
$$

then the call price can be expressed in the form

$$
C_{0}=P_{0 t} \mathbb{E}\left[\frac{1}{\Phi_{t}}\left(\sum_{i} \sum_{k}\left(P_{t T} x_{i}-K\right) p_{i k t}\right)^{+}\right] .
$$

Our strategy now is to eliminate the term $\Phi_{t}^{-1}$ via a measure change technique.

We remark first that it follows from (25) and (30) that

$$
\mathrm{d} \Phi_{t}=\left(\frac{T}{(T-t)} \mathbb{E}\left[\sigma X_{T} \mid \xi_{t}\right]\right)^{2} \Phi_{t} \mathrm{~d} t+\frac{T}{T-t} \mathbb{E}\left[\sigma X_{T} \mid \xi_{t}\right] \Phi_{t} \mathrm{~d} W_{t}
$$

from which it follows that

$$
\mathrm{d} \Phi_{t}^{-1}=-\frac{T}{T-t} \mathbb{E}\left[\sigma X_{T} \mid \xi_{t}\right] \Phi_{t}^{-1} \mathrm{~d} W_{t} .
$$

Expressed in an integral form, we thus have

$$
\Phi_{t}^{-1}=\exp \left(-\int_{0}^{t} \frac{T}{T-s} \mathbb{E}\left[\sigma X_{T} \mid \xi_{s}\right] \mathrm{d} W_{s}-\frac{1}{2} \int_{0}^{t} \frac{T^{2}}{(T-s)^{2}} \mathbb{E}\left[\sigma X_{T} \mid \xi_{s}\right]^{2} \mathrm{~d} s\right) .
$$

Since $\mathbb{E}\left[\sigma X_{T} \mid \xi_{t}\right]$ is bounded, the Novikov condition

$$
\mathbb{E}\left[\exp \left(\frac{1}{2} \int_{0}^{t} \frac{T^{2}}{(T-s)^{2}} \mathbb{E}\left[\sigma X_{T} \mid \xi_{s}\right]^{2} \mathrm{~d} s\right)\right]<\infty
$$

is satisfied. Hence $\left\{\Phi_{t}^{-1}\right\}_{0 \leq t \leq u<T}$ is a martingale. We also deduce that

$$
\Phi_{0}^{-1}=\left(\sum_{i} \sum_{k} p_{i} q_{k}\right)^{-1}=1
$$

and hence that $\mathbb{E}\left[\Phi_{t}^{-1}\right]=1$. Thus the factor $\Phi_{t}^{-1}$ can be used to effect a change of measure. Writing $\mathbb{B}_{T}$ for the new measure, the option price then becomes:

$$
C_{0}=P_{0 t} \mathbb{E}^{\mathbb{B}_{T}}\left[\left(\sum_{i} \sum_{k}\left(P_{t T}-K\right) p_{i k t}\right)^{+}\right]
$$


It is not difficult to show that under $\mathbb{B}_{T}$, the information process $\left\{\xi_{s}\right\}_{0 \leq s \leq t}$ is Gaussian with mean 0 and variance $t(T-t) / T$; that is to say, $\left\{\xi_{t}\right\}$ is a $\mathbb{B}_{T}$-Brownian bridge. The Radon-Nikodým derivative associated with this measure change is thus $d \mathbb{B}_{T}=\Phi_{t}^{-1} \mathrm{~d} \mathbb{Q}$. The random variables $X_{T}$ and $\sigma$ have the same probability law with respect to $\mathbb{B}_{T}$ as with $\mathbb{Q}$, and the conditional expectation of any integrable function $f\left(X_{T}, \sigma\right)$ can be expressed as:

$$
\mathbb{E}^{\mathbb{B}_{T}}\left[f\left(X_{T}, \sigma\right) \mid \mathcal{F}_{t}\right]=\frac{\mathbb{E}^{\mathbb{Q}}\left[\Phi_{t}^{-1} f\left(X_{T}, \sigma\right) \mid \mathcal{F}_{t}\right]}{\mathbb{E}^{\mathbb{Q}}\left[\Phi_{t}^{-1} \mid \mathcal{F}_{t}\right]}
$$

In particular, the process $\left\{W_{t}^{*}\right\}_{0 \leq t \leq u}$ defined by

$$
W_{t}^{*}=\int_{0}^{t} \frac{T}{T-s} \mathbb{E}\left[\sigma X_{T} \mid \xi_{s}\right] \mathrm{d} s+W_{t}
$$

is a $\mathbb{B}_{T}$ Brownian motion. To verify that $\left\{\xi_{t}\right\}$ is a $\mathbb{B}_{T}$-Brownian bridge, we substitute (30) in (56) to deduce

$$
\mathrm{d} \xi_{t}=-\frac{\xi_{t}}{T-t} \mathrm{~d} t+\mathrm{d} W_{t}^{*}
$$

But this is just the SDE for a Brownian bridge process in the $\mathbb{B}_{T}$ measure.

Returning to the problem of option pricing, let us begin by considering the case of a binary bond whereby the cash flow takes the two possible values $\left\{x_{0}, x_{1}\right\}$. Then we have

$$
C_{0}=P_{0 t} \mathbb{E}^{\mathbb{B}_{T}}\left[\left(\left(P_{t T} x_{1}-K\right) \sum_{k} p_{1 k t}+\left(P_{t T} x_{0}-K\right) \sum_{k} p_{0 k t}\right)^{+}\right] .
$$

This expectation is nontrivial when $P_{t T} x_{1}>K>P_{t T} x_{0}$. In this case, the option can expire either in the money or out of the money, depending on whether $\xi_{t}>\xi^{*}$ or $\xi_{t}<\xi^{*}$, where $\xi^{*}$ is the unique critical value of $\xi_{t}$ such that $B_{t} T=K$, or, equivalently, the unique solution to the relation

$$
\frac{\sum_{k} q_{k} \exp \left(\frac{T}{T-t}\left(\sigma_{k} x_{0} \xi^{*}-\frac{1}{2} \sigma_{k}^{2} x_{0}^{2} t\right)\right)}{\sum_{k} q_{k} \exp \left(\frac{T}{T-t}\left(\sigma_{k} x_{1} \xi^{*}-\frac{1}{2} \sigma_{k}^{2} x_{1}^{2} t\right)\right)}=\frac{p_{1}\left(K-P_{t T} x_{1}\right)}{p_{0}\left(P_{t T} x_{0}-K\right)} .
$$

Note that in general $\xi^{*}$ has no closed-form expression. However, the solution to (59) can be obtained by simple numerical root-finding methods. That the solution to (59) is unique (assuming that $\sigma$ is a positive random variable) can be seen by the fact that the bond price is an increasing function of $\xi_{t}$.

The problem of option pricing thus reduces to performing an elementary Gaussian integration. We now consider the case where $X_{T}$ need not be a binary variable. The computation simplifies further if we introduce a standard normal variable $Z$ according to

$$
Z=\frac{\xi_{t}}{\sqrt{t(T-t) / T}}
$$

We write $Z^{*}$ for the corresponding critical value. Then the option pricing formula is:

$$
C_{0}=P_{0 t} \sum_{k} \sum_{i} q_{k} p_{i}\left(P_{t T} x_{i}-K\right) N\left(\sqrt{\tau} \sigma_{k} x_{i}-Z^{*}\right)
$$




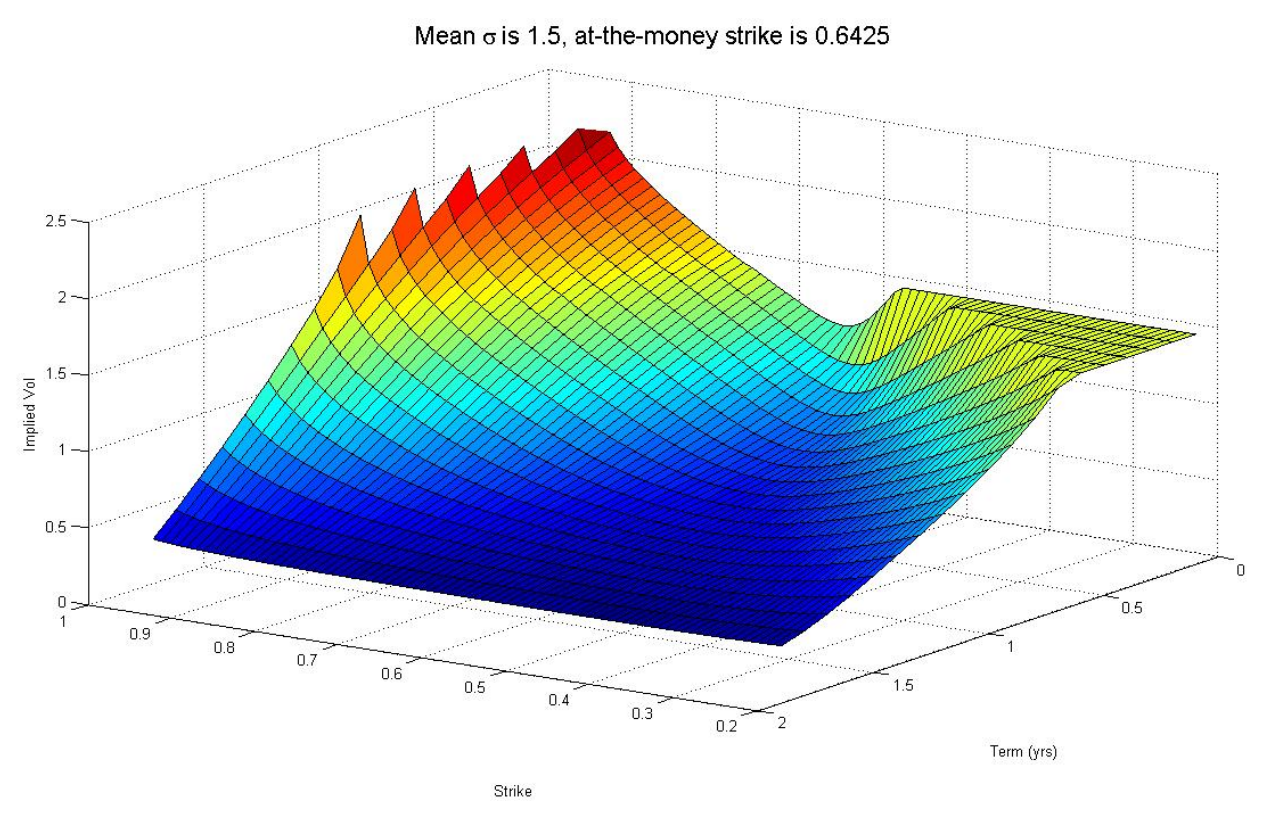

FIG. 7: The implied BHM volatility. The implied volatility surface resulted from calibrating each strike and maturity of the random information model to the BHM model is shown. The parameters are set to be $X_{T}=\{0,1\}, p_{i}=\{0.2,0.8\}, \sigma_{\mathrm{BHM}}=1.5, \sigma_{\text {Rand }}=\{0.3,2.7\}, q_{k}=\{0.5,0.5\}, r=0 \%$ and $T \in[0,2]$.

where $\tau=t T /(T-t)$ and $N(\cdot)$ denotes the standard cumulative normal density function.

Remark: We have noted that our random information model can be viewed as a mixture of BHM models. In the context of the Black-Scholes model, Renault and Touzi [15] or Brigo et al. [16], for instance, have carried out similar analysis, where the Black-Scholes volatility parameter $\sigma$ is taken to be time dependent random variable that is independent of the underlying Brownian motion. By conditioning on the volatility path, the European call option is obtained as the expectation of the Black-Scholes call price with time-averaged volatility. In particular, when $\sigma$ is time independent, the result of $[15,16]$ for the extended Black-Scholes model is similar in nature to our result on extended BHM model.

Remark: The randomisation of the Black-Scholes volatility parameter (the log-normal mixture) in the literature is carried out essentially in an ad hoc manner, without any fundamental economic or information-theoretic reason. Rather, it is justified on the practical ground that it gives a better handling of calibration. In contrast, in our model the randomisation arises from a more realistic analysis on the market information process. Hence, although the net effect is similar in both cases, our model is accompanied by a better justification, which in turn also gives a better justification for the lognormal mixture models. In particular, it can be seen, by applying the present scheme on the information-based derivation of the Black-Scholes model in [4], that the lognormal mixture models can be derived by assuming that the information flow-rate is random.

The random information-flow model considered here can be viewed as the simplest stochastic volatility model for option pricing in the information based asset pricing framework. One natural and important question arising in the present model is: how do we calibrate the distribution of $\sigma$ ? The answer is given by the volatility surface. We note that in the original BHM model for defaultable bonds the information flow rate parameter 

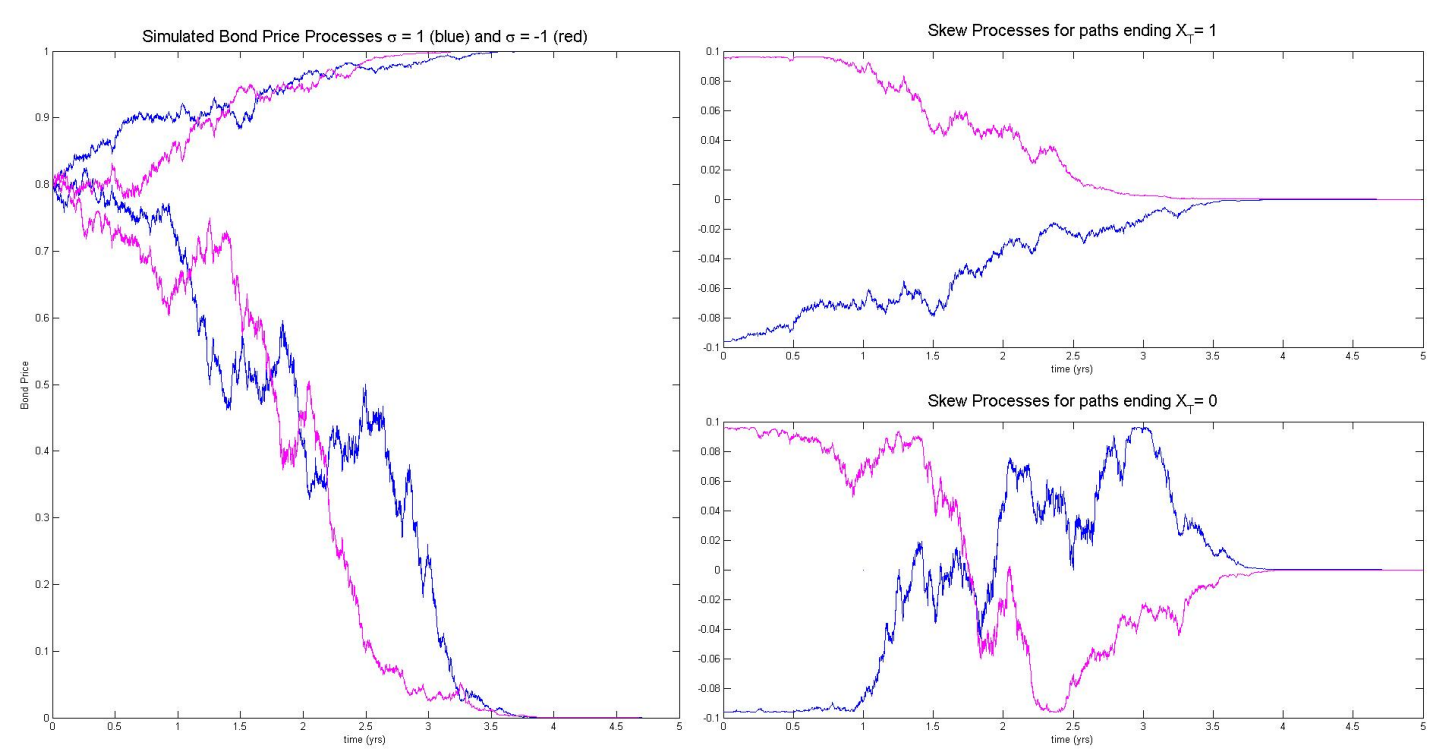

FIG. 8: Left: Sample paths of the BHM with $\sigma=1$ (blue) and $\sigma=-1$ (red) using same Brownian Bridges; and the corresponding skew processes associated with $X_{T}(\omega)=1$ (Top right) and $X_{T}(\omega)=$ 1 (Bottom right). Here we set $X_{T}=\{0,1\}, p_{i}=\{0.2,0.8\}, r=0 \%$ and $T=5$.

is calibrated by the option price for a fixed strike and a fixed maturity. Hence the model cannot be used to calibrate against the volatility surface. In contrast, the random- $\sigma$ model considered here has a wider flexibility that allows for the calibration of larger market data set. To illustrate the idea, we have plotted in figure 7 the option price (61) in our random- $\sigma$ model, but expressed in the form of an implied BHM volatility surface.

\section{INFORMATION MANIPULATION}

We conclude by drawing attention to another interesting feature of the variable $\sigma$ model. This concerns the notion of information manipulation, or, equivalently, a deliberate misrepresentation of information. The question that we are interested in here is the following: How does one model the manipulation of information in the information-based framework? One possible solution that we examine here is based on the misspecification of the information flow-rate $\sigma$.

The idea can be sketched as follows. Each market agent reveals information, expressed in the form of one of the information processes of (3). The impact of that agent's information on the market is then determined through formula (4). If an agent releases information that is based purely on speculation, then that information source is noise dominated, having a small value of the information flow rate. However, if that agent is trying to mislead the market, based on a reliable piece of information, then that information source is no longer noise dominated. Instead, this misinformation can be modelled by the fact that the agent provides an incorrect value for the information flow rate parameter. In this way, the market will estimate the 'fair' price of the asset - in the present example the defaultable bond - by use of the pricing formula (16), but based on the incorrect value for the information flow rate parameter. As a consequence, the market price will be misled.

As an illustration of this behaviour, in figure 8 we show sample paths for the defaultable 
digital bond price process; in one case where the bond did not default, whereas in the other case where the bond defaulted. In each case, two sample paths are given; one corresponding to the situation where the true market price ought to be if there is no misleading information, and one corresponding to the situation where the realised price is, due to the existence of a deliberate price manipulation. In order to make the effect of price manipulation visually pronounced, here we have taken a slightly extreme case in which the true value of the information flow rate is $\sigma=+1$ (blue), whereas the 'conjugate' price process is generated by the false belief that the flow rate is given by $\sigma=-1$ (red). We find in these examples the existence of a kind of anti-correlation between the 'true' and 'false' price movements around their conditional means. This is illustrate more clearly in the skewness plot, also shown in figure 8 .

One might enquire in which way a mis-specification of the information flow rate $\sigma$ is realised in practice. In this connection it is worth remarking that there is an extended literature on price manipulation, often in the context of insider trading. One typical way of spreading a false information is by taking a trading position in a strategic manner (cf. $[17,18])$. For example, suppose that an informed trader has the information that the price of an asset is likely to drop in near future. In this case, taking a short position amounts to effectively revealing the content of that information. Hence, by momentarily taking a long position before taking a short position, an informed trader can mislead the market. One can think of such a deliberate manoeuvre being represented abstractly in the form of one of the information processes in (3) taking an 'incorrect' value of $\sigma$.

We see therefore that the information-based framework allows for a range of flexible extensions to model various scenarios that might occur in a given financial market. It would be of considerable interest, in particular, to develop further the information-based approach to price manipulation under the present scheme.

\section{Acknowledgments}

The authors thank M. H. A. Davis and L. P. Hughston for comments and stimulating discussion. YTL thanks HSBC for support.

[1] Brody, D. C., Hughston, L. P. and Macrina, A. 2007 "Beyond hazard rates: a new framework for credit-risk modelling" Advances in Mathematical Finance: Festschrift Volume in Honour of Dilip Madan (Basel: Birkhäuser).

[2] Brody, D. C., Hughston, L. P. and Macrina, A. 2008 "Information-based asset pricing" International Journal of Theoretical and Applied finance 11, 107-142.

[3] Rutkowski, M. and Yu, N. 2007 "On the Brody-Hughston-Macrina approach to modelling of defaultable term structure" International Journal of Theoretical and Applied finance 10, 557-589.

[4] Brody, D.C., Hughston, L.P. \& Macrina, A. (2011) "Modelling information flows in financial markets" In Advanced Mathematical Methods for Finance, p. 133-153, G. Di Nunno \& B. Øksendal, eds. (Berlin: Springer).

[5] Grossman, S. J. \& Stiglitz, J. E. (1980) "On the impossibility of informationally efficient markets" The American Economic Review 70 393-408. 
[6] Grossman, S. J. 2003 The Informational Role of Prices, 2nd revised ed. (Boston: The MIT Press).

[7] Anderson, T., Bollerslev, T., Diebold, F. X. and Vega, C. 2007 "Real-time price discovery in stock, bond and foreign exchange markets" Journal of International Economics 73 251-277.

[8] Bollen, J., Mao, H. \& Zeng, X.-J. 2011 "Twitter mood predicts the stock market" Journal of Computational Science, 2, 1-8.

[9] Brody, D.C., Meister, B. K. \& Parry M.F. 2012 "Informational inefficiencies in financial markets" Mathematics and Financial Economics 6, 249-259.

[10] Brody, D. C., Davis, M. H. A., Friedman, R. L. and Hughston, L. P. 2009 "Informed traders" Proceedings of Royal Society A465 11031122.

[11] Fisher, R. A. 1925 "Theory of statistical estimation" Proceedings of the Cambridge Philosophical Society 22, 700-725.

[12] Rao, C. R. 1945 "Information and accuracy attainable in the estimation of statistical parameters" Bulletin of the Calcutta Mathematical Society 37, 81-91.

[13] Gel'fand, I. M. and Yaglom, A. M. 1957 "Calculation of the amount of information about a random function contained in another such function" Uspekhi Matematicheskikh Nauk 12, $3-52$.

[14] Khintchine, A. Ya. 1953 "The concept of entropy in the theory of probability" Uspekhi Matematicheskikh Nauk 8, 3-20.

[15] Renault, E. and Touzi, N. 1996 "Option hedging and implied volatilities in a stochastic volatility model" Mathematical Finance 6, 279302.

[16] Brigo, D., Mercurio, F. and Rapisarda, F. 2004 "Smile at the uncertainty" Risk Magazine May, 97-101.

[17] Easley, D. and O'Hara, M. 1987 "Price, trade, size, and information in securities markets" Journal of Financial Economics 19, 69-90.

[18] Allen, F. and Gale, D. 1992 "Stock-price manipulation" Review of Financial Studies 5, 503529. 